\title{
Aguas Profundas, un Efecto en la Temperatura para el Manejo de Caligidosis en el Salmón del Atlántico (Salmo salar)
}

\author{
DeEP W ATER, AN EFfect ON THE Temperature For the Management OF \\ Caligodosis in the Atlantic Salmon (Salmon Salar)
}
Roberto Riquelme ${ }^{1, *}$, Pamela Olivares-Ferretti ${ }^{1, *}$, Flery Fonseca-Salamanca ${ }^{2}$, Jorge Parodi ${ }^{1,3}$

\section{Resumen}

\begin{abstract}
La salmonicultura constituye uno de los pilares de la economía chilena, pero debido al desarrollo de innumerables enfermedades, entre las que destaca la ectoparasitosis causada por Caligus rogercresseyi, los productores de salmón han disminuido sus índices productivos. Debido a esto, se están buscando sistemas alternos para cultivar salmones, siendo uno de ellos el cultivo de peces en aguas profundas, donde la temperatura es menor que la temperatura de la superficie, ya que $C$. rogercresseyi es un parásito cuyo ciclo de vida es dependiente de la temperatura del agua. El estudio detalla el comportamiento biológico del parásito y el efecto de la profundidad y temperatura de cultivo sobre el establecimiento del parásito en el hospedero.
\end{abstract}

Palabras clave: jaulas «snorkel», temperatura, Caligus rogercresseyi

\section{AbstraCt}

Salmon farming is one of the pillars of the Chilean economy but due the emerging of many diseases, including the ecto-parasitism caused by Caligus rogercresseyi, the salmon industry has decreased their production indices. Based on that, alternative rearing systems are being evaluated for salmon cultivation, one of them fish farming in deep water, where

\footnotetext{
${ }^{1}$ Laboratorio de Biología Celular Aplicada, Núcleo de investigación en Producción Alimentaria-NIPA, Facultad de Recursos Naturales, Universidad Católica de Teтuco, Teтuco, Chile

${ }^{2}$ Laboratorio de Inmuno Parasitología Molecular, Centro de Excelencia en Medicina Traslacional, Departamento de Ciencias Preclínicas, Universidad de La Frontera, Temuco, Chile

${ }^{3}$ E-mail:jparodi@uct.cl

* Ambos autores colaboraron de forma igual en el manuscrito
} 
the temperature is lower than the temperature of the surface, as C. rogercresseyi is a parasite whose life cycle is water temperature dependent. This paper describes the biological behaviour of the parasite and the effect of deep water and culture temperature on the establishment of the parasite in the host.

Key words: «snorkel» cages, temperature, Caligus rogercresseyi

\section{INTRODUCCIÓN}

Desde comienzos de la década de los ochenta, Chile ha experimentado y desarrollado un notable y sostenido crecimiento en la industria de producción de salmónidos en cautiverio, posicionándose como uno de los principales países productores a nivel mundial (Osorio, 2006). Al inicio de la salmonicultura, las condiciones sanitarias para el cultivo eran bastante beneficiosas, pero a medida que los sistemas productivos se fueron intensificando, las enfermedades manifestaron un significativo aumento. Uno de los problemas sanitarios ha sido la diseminación de ectoparásitos, específicamente del crustáceo copépodo Caligus rogercresseyi (Gonzáles y Carvajal, 2003).

Este parásito, Ilamado comúnmente «piojo de mar», habita las aguas marinas y salobres de Chile, parasitando a las salmonicul turas y, de esa forma, generando pérdidas económicas, tanto por la disminución del crecimiento de los peces como por el costo de los tratamientos aplicados para mitigar el problema (Bravo, 2010; Bravo et al., 2014).). El tratamiento de este parásito trae como consecuencia el alargamiento de los ciclos productivos, debido a que disminuye la ingesta de alimento, predisponiendo a los peces a infecciones secundarias (Gajardo, 2011).

La existencia de caligidosis en Chile data del año 1940. Se reporta que, a diferencia de su homólogo Lepeophtheirus salmonis, que parasita peces silvestres en el hemisferio norte, $C$. rogercresseyi es un copépodo natural de los peces marinos del hemisferio sur, entre los que se puede mencionar Zearaja chilensis (raya), Merluccius australis (merluza), Paralichthys californicus (lenguado), Eleginops maclovinus (róbalo) y Odontesthes bonariensis (pejerrey) (Bravo et al., 2015b). Oncorhynchus kisutch (salmón coho) pareciera indicar que es la especie más resistente al copépodo $L$. salmonis, teniendo significativamente menos copepoditos que el Oncorhynchus tshawytscha (salmón chinook) o Salmo salar (s. del Atlántico) entre los 15 y 20 días de exposición (Jonhson y Albright, 1992). Por otro lado, se ha demostrado que Oncorhynchus mykiss (trucha arcoíris) es más susceptible que $O$. kisutch y $S$. salar (González et al., 2000).

El asentamiento de esta parasitosis está ligada a una gran variedad de factores ambientales, biológicos y de manejo, que influyen y convergen para que se desarrolle la infestación. Dentro de estos parámetros, la temperatura tiene un rol de gran importancia en el desarrollo del ciclo de este copépodo (Bravo, 2010; Yatabe et al., 2011), ya que incide directamente sobre la velocidad y viabilidad de sus procesos fisiológicos. Se indica que su ciclo de vida depende directamente de la temperatura del agua; así, en la trucha arco iris el ciclo de vida dura 45 días a una temperatura de $10.3{ }^{\circ} \mathrm{C}, 32$ días a $12.8{ }^{\circ} \mathrm{C}$ y 26 días a $15.2^{\circ} \mathrm{C}$ (Bravo et al., 2013). Además, se ha estimado que el umbral mínimo de temperatura para el desarrollo del parásito es $4.2^{\circ} \mathrm{C}$ (Gonzáles y Carvajal, 2003), ya que a menores temperaturas el copépodo disminuye el consumo de oxígeno y alimento (Fernández, 1978). 
Es por ello que en este trabajo se ha pretendido esclarecer la factibilidad de cultivar salmones a menor temperatura y a mayor profundidad, sin afectar los índices productivos de los peces, con el fin de disminuir el contagio de Caligus rogercresseyi.

\section{Enfermedades en Peces}

Como todo animal, los peces son susceptibles a las enfermedades, las que se presentan tanto en la vida silvestre como en la producción en cautiverio (Vanderstichel et al., 2014). Las enfermedades presentan una mayor incidencia en los centros de producción en comparación con las cuencas hídricas naturales (Figura 1), a consecuencia de las altas densidades poblacionales requeridas para su crianza comercial. Las enfermedades generan pérdidas económicas importantes, siendo responsables de mortalidades masivas en la explotación, sobre todo en las fases de cría y alevín (González et al., 2001).

Las enfermedades causadas por bacterias, virus y hongos son de gran importancia en la industria del salmón, y en forma similar, los parásitos son causantes de diversas enfermedades (Rodríguez et al., 2001). La parasitosis más importante y de mayor trascendencia que ha generado grandes preocupaciones a los países productores de salmón, es la caligidosis, causada por el ectoparásito Caligus rogercresseyi (Bravo et al., 2015b).

\section{Ciclo de Vida de $C$. rogercresseyi}

Presenta ocho etapas de desarrollo (Figura 2): tres planctónicas y cinco parasitarias (Bravo et al., 2015b). Las etapas planctónicas comprenden dos napulios y un copepodito que corresponde a la fase infectante. En la fase planctónica, el promedio de longitud es de $0.43 \mathrm{~mm}$ para el napulio I, de $0.46 \mathrm{~mm}$ para el napulio II y de 0.66 $\mathrm{mm}$ en el estado de copepodito. En las fases parasitarias, en la etapa chalimus I se registra una longitud de $0.83 \mathrm{~mm}$, chalimus II de $1.27 \mathrm{~mm}$, chalimus III de $2.15 \mathrm{~mm}$ y chalimus
IV de $3.15 \mathrm{~mm}$. El macho adulto llega a tener una longitud promedio de $4.83 \mathrm{~mm}$ y la hembra adulta con huevos de $4.79 \mathrm{~mm}$ (Gonzáles y Carvajal, 2003).

En el estado adulto se presenta un marcado dimorfismo sexual, donde el complejo genital en machos es más redondo y en forma de barril, mientras que en la hembra es más cuadrado y con presencia de puntas lateroposteriores (Bravo, 2010). El comportamiento de apareamiento es esencial para la supervivencia del parásito. La búsqueda de la pareja se lleva a cabo por $C$. rogercresseyi adulto en presencia de la hembra adulta. Este comportamiento proporciona evidencias que el reconocimiento de la pareja puede ocurrir por señales químicas o por contactos (Ritchie et al., 1996; Lonsdale et al., 1998). Los procesos de comportamiento, las señales químicas y la cópula se encuentran ampliamente descritos para $L$. salmonis, mas no así en $C$. rogercresseyi.

C. rogercresseyi, como copepodito, se adhiere al hospedero utilizando sus antenas modificadas como gancho (Farías, 2005). Una vez adherido, desarrolla el filamento frontal, generado por la glándula de cemento ubicada en la parte anterior del cefalotórax, el que penetra en la piel del pez permitiéndole una mayor adhesión (Osorio, 2006). Una vez instalado, comienzan las cuatro etapas parasitarias de chalimus, que se desarrollan mientras el parásito permanece fuertemente unido al pez, por medio del filamento frontal, hasta llegar al estado adulto. Tanto machos como hembras parasitan al hospedero alimentándose de células mucosas, células pigmentadas y glándulas mucosas.

La fijación y la alimentación del parásito sobre el pez ocasionan una disminución de la barrera mucosa (Bravo et al., 2013), ya que el parásito se alimenta de las glándulas generadoras del mucus, inhibiendo su producción; asimismo, al consumir la epidermis, desaparecen las células de la capa germinativa, que son las que se especializan en regenerar células muertas o erosionadas de la piel 

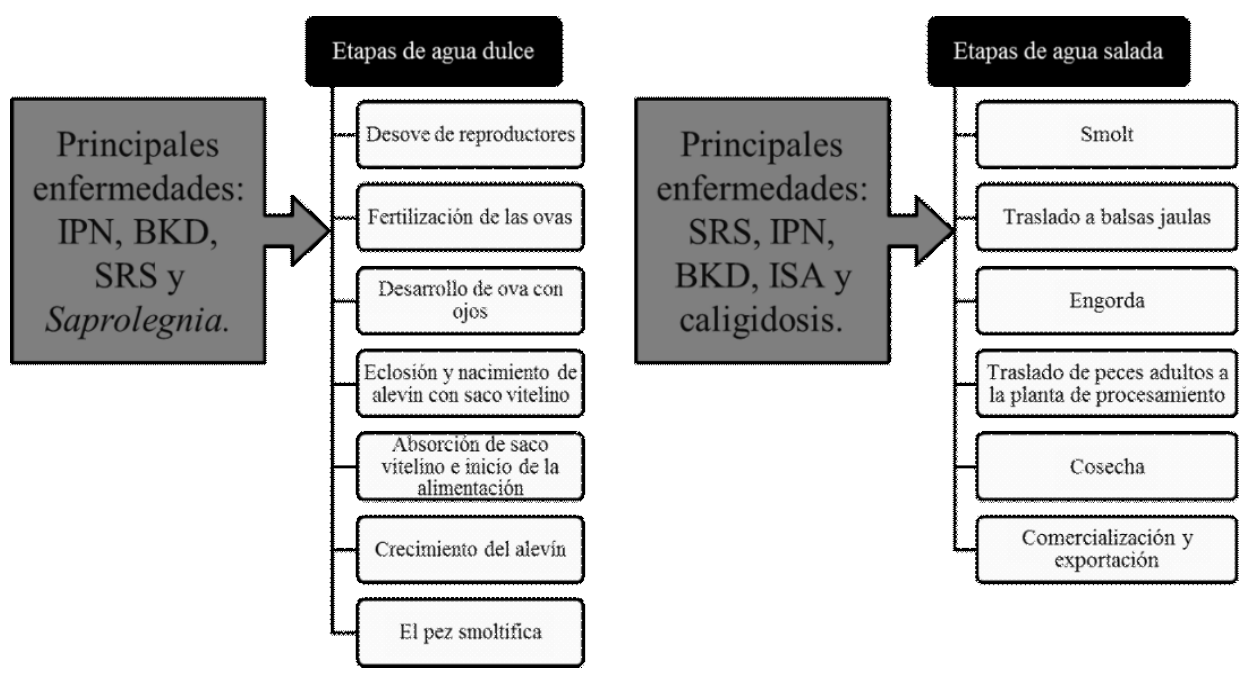

Figura 1. Enfermedades que alteran a la salmonicultura en etapas de agua dulce y agua salada, según la fase del ciclo de desarrollo

(Osorio, 2006). Como consecuencia de este daño, el hospedero presenta pérdida de epitelio, úlceras, descarga mucosa aumentada para compensar la pérdida ocasionada por la parasitosis, alteración de la bioquímica del mucus, necrosis tisular y la consecuente pérdida de función de la piel como barrera física y microbiológica (Bravo et al., 2014). De este modo, los peces afectados reducen su apetito, crecimiento y eficiencia de conversión alimentaria, mientras que el estrés y las lesiones producidas por el parásito los hacen más susceptibles de infecciones secundarias, producto de una inmunocompetencia disminuida (Bravo et al., 2015a,b).

\section{Cultivo y Temperatura}

En los cultivos de salmones en Chile, durante la etapa de engorda, los peces son trasladados al mar, donde son colocados en balsas jaulas, que varían de tamaño y a diferentes profundidades. El tipo de jaula más comúnmente usado es el que se ubica entre 1 y $5 \mathrm{~m}$ de profundidad, ancladas al fondo marino a bloques de concreto (Nieto et al., 2010).
Los cultivos de salmones se ubican en la primera capa de aguas marinas, donde las temperaturas fluctúan entre los 8 y $13{ }^{\circ} \mathrm{C}$ (Solis, 2009). En estos ambientes, la temperatura del agua disminuye conforme aumenta la profundidad (SHOA, 2015).

S. salar es una especie anádroma, que habitaba exclusivamente en el hemisferio norte (Solis, 2009), pero a partir de $1870 \mathrm{y}$ por más de 60 años, Estados Unidos impulsó la introducción de ovas de $S$. salar a países del hemisferio sur, siendo Chile el principal destino (Silva, 2007). Chile posee grandes ventajas para la producción de salmones, ya que la zona sur austral y principalmente, entre las X y XI regiones, los factores ambientales se ajustan perfectamente a sus requerimientos fisiológicos como temperatura, $\mathrm{pH}$, oxígeno, salinidad y pureza de las aguas (Nieto et al., 2010). Los salmónidos son seres poiquilotermos; es decir, que dependen de la temperatura ambiental (Fernández et al., 2002). Crecen y se desarrollan entre 6 y $16^{\circ} \mathrm{C}$ y salinidades que fluctúan en niveles de 28 a 32 ppm (Bravo et al., 2015a), entre otros factores. Por esta razón, la etapa de engorda en 


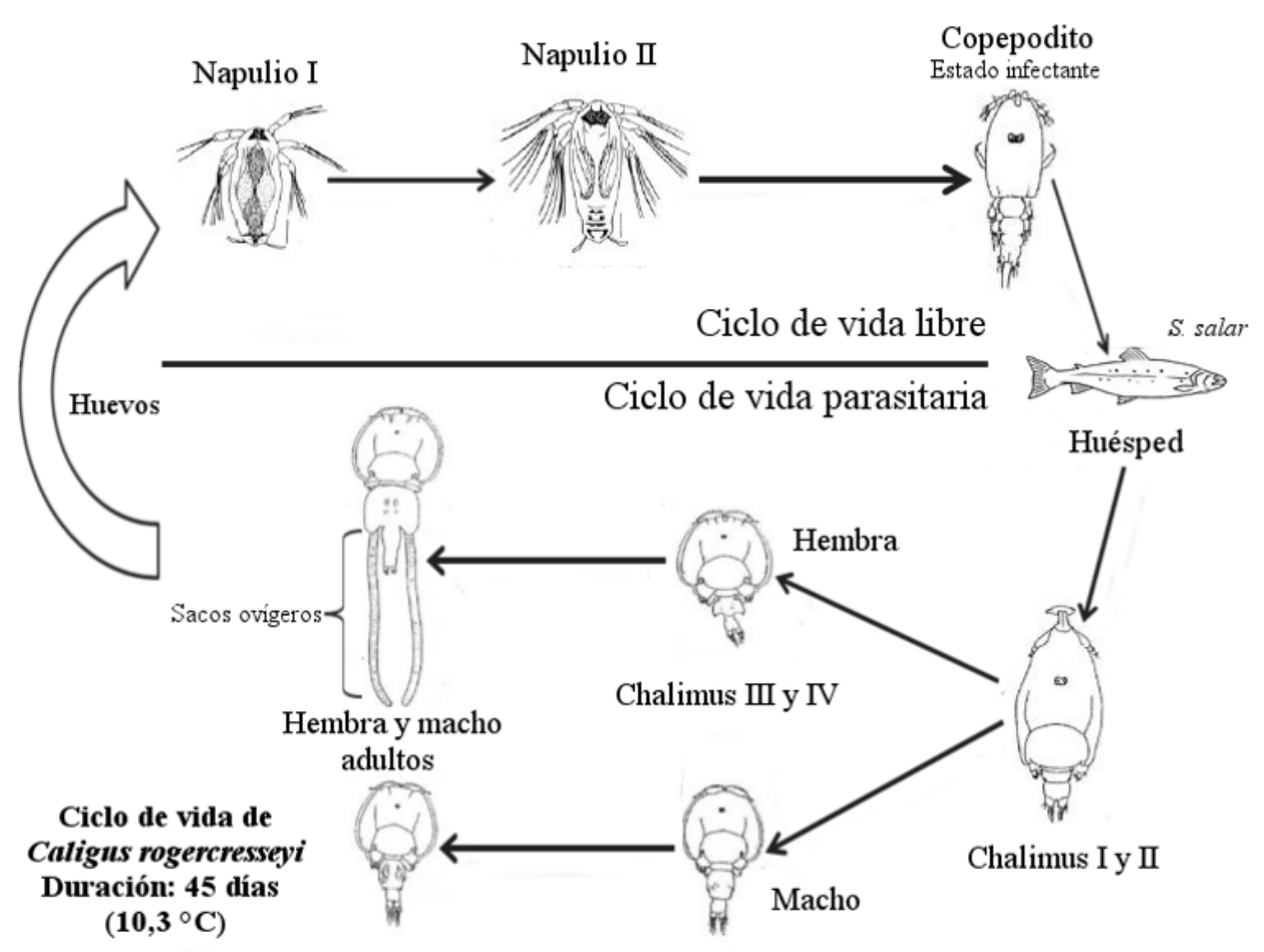

Figura 2. Ciclo de vida de Caligus rogercresseyi. Duración: 45 días a $10.3{ }^{\circ} \mathrm{C}$

el cultivo del salmón se desarrolla en centros ubicados en el mar (Flores y Vergara, 2012).

La temperatura del agua es fundamental dentro de la fisiología de los peces. Su incremento aumenta el metabolismo y, en consecuencia, los requerimientos energéticos que se deben satisfacer mediante el consumo de alimento (Fernández et al., 2002). La temperatura, además, influye en su capacidad reproductiva; así la temperatura óptima para $O$. mykiss es de $15{ }^{\circ} \mathrm{C}$, en tanto que para Cyprinus carpio (carpa) es superior a $20{ }^{\circ} \mathrm{C}$. Por otro lado, la temperatura ejerce un profundo efecto sobre la tasa de desarrollo de las larvas y la supervivencia de los embriones (Hyeland et al., 2008). Diversos estudios muestran que la temperatura acelera la miogénesis, así como el desarrollo de órganos y tejidos corporales de varias espe- cies de teleósteos, incluyendo $S$. salar (Flores y Vergara, 2012). La temperatura es un factor que afecta el metabolismo respiratorio y la excreción de amonio en las especies acuáticas; asimismo, el consumo de $\mathrm{O}_{2}$ y la excreción de $\mathrm{N}$ total se incrementan con el aumento de la temperatura (Nytro et al., 2013).

La temperatura, vista desde otro enfoque, desempeña un rol fundamental en el funcionamiento de un ecosistema, al regular o afectar los componentes que determinan el espacio físico en el cual habitan los individuos; influyendo en la solubilidad de nutrientes, gases, estado físico de los nutrientes, grado de toxicidad y propiedades físico-químicas del medio acuoso (Wijekoon et al., 2014). 


\section{Protocolos de Cultivo en Aguas Profun- das}

Estudios realizados en Noruega con $L$. salmonis demuestran que los copepoditos se encuentran, usualmente, en los primeros 3 a $4 \mathrm{~m}$ de la columna de agua (Bricknell et al., 2006); pudiendo inferirse que los peces que habitan en aguas superficiales presentarían una mayor predisposición a enfermar que aquellos que habitan en aguas más profundas (Bravo, 2010), de allí que el cultivo de salmones en aguas más profundas que las normalmente utilizadas podría disminuir el contagio y la carga parasitaria de $C$. rogercresseyi en el salmón.

Dentro de los sistemas de cultivo en aguas profundas están las jaulas tipo snorkel (Figura 3), que se ubican a 3 o 4 metros de profundidad, y por donde se puede acceder a la superficie mediante un tubo central, que le da el nombre de snorkel. El tubo central, hecho de lona o red de plancton, impide el paso de los copepoditos y permite generar un área libre de parásitos (Stien et al., 2015). De esta forma, los peces pueden llegar a la superficie y llenar de aire su vejiga natatoria, lo que convierte a estas jaulas en una buena opción en comparación con otras jaulas totalmente sumergidas, donde los peces no tienen acceso a la superficie (Føre et al., 2011), de allí que se desencadena una serie de efectos negativos sobre el crecimiento y comportamiento de los peces.

La eficacia de cultivos jaulas tipo snorkel, a diferencia de cultivos en balsas jaulas ubicadas en aguas superficiales, se encuentra entre 24 y $65 \%$ de disminución del contagio por el copépodo, según el lugar de cultivo y los niveles de contaminación con formas libres del parasito. Por otro lado, las tasas de crecimiento o el bienestar de los peces se ve alterado en las jaulas snorkel (Stien et al., 2015).

El otro sistema de cultivo de peces en aguas profundas está referido al uso de jaulas totalmente sumergidas, que restringen el libre acceso del pez a la superficie (Figura 4) (Oppedal et al., 2016). Se describe que los salmones pueden tolerar la inmersión hasta 4 $m$ de profundidad durante tres semanas, sin presentar alteraciones en el crecimiento y bienestar (Dempster et al., 2008). Este tipo de cultivo presentaría una serie de ventajas ya que se reducirían los daños por tormentas, floraciones de medusas y algas, e infestaciones por $C$. rogercresseyi, entre otros; pero presenta desventajas, ya que, al prolongarse el tiempo de inmersión, los peces presentan disminución del crecimiento, alteración en los patrones de natación y, por lo tanto, un déficit en su bienestar. Esto es debido a una alteración en la flotabilidad, ya que el pez no tiene acceso a la superficie para llenar su vejiga natatoria (Korsøen et al., 2011).

\section{Eficiencia en el Control de Caligidosis Cultivando Salmones en Aguas Profun- das}

Este tipo de cultivo parece ser prometedor por las evidencias experimentales en otros países. En Chile se están iniciando estos estudios estándose a la espera de datos de producción concretos para validarlo en los modelos locales de cultivo. Estudios experimentales en cultivo de $S$. salar con jaulas tipo snorkel muestran una reducción importante de piojos cuando los peces nadan en aguas profundas a menores temperaturas (Stien et al., 2015; Oppedal et al., 2016). Asimismo, peces que nadan a 12 o más metros de profundidad en los meses de invierno reducen la infección por $C$. rogercresseyi prácticamente a cero. En 2015, utilizando jaulas snorkel a escala comercial, se reportaron reducciones mayores de $65 \%$ de parásitos en los peces, comparado con jaulas normales dentro de la misma zona (Oppedal et al., 2016). Las jaulas de cultivo tipo snorkel han demostrado ser una solución innovadora, sostenible y respetuosa con el medio ambiente donde, además, los índices productivos del salmón y su bienestar están salvaguardados. 


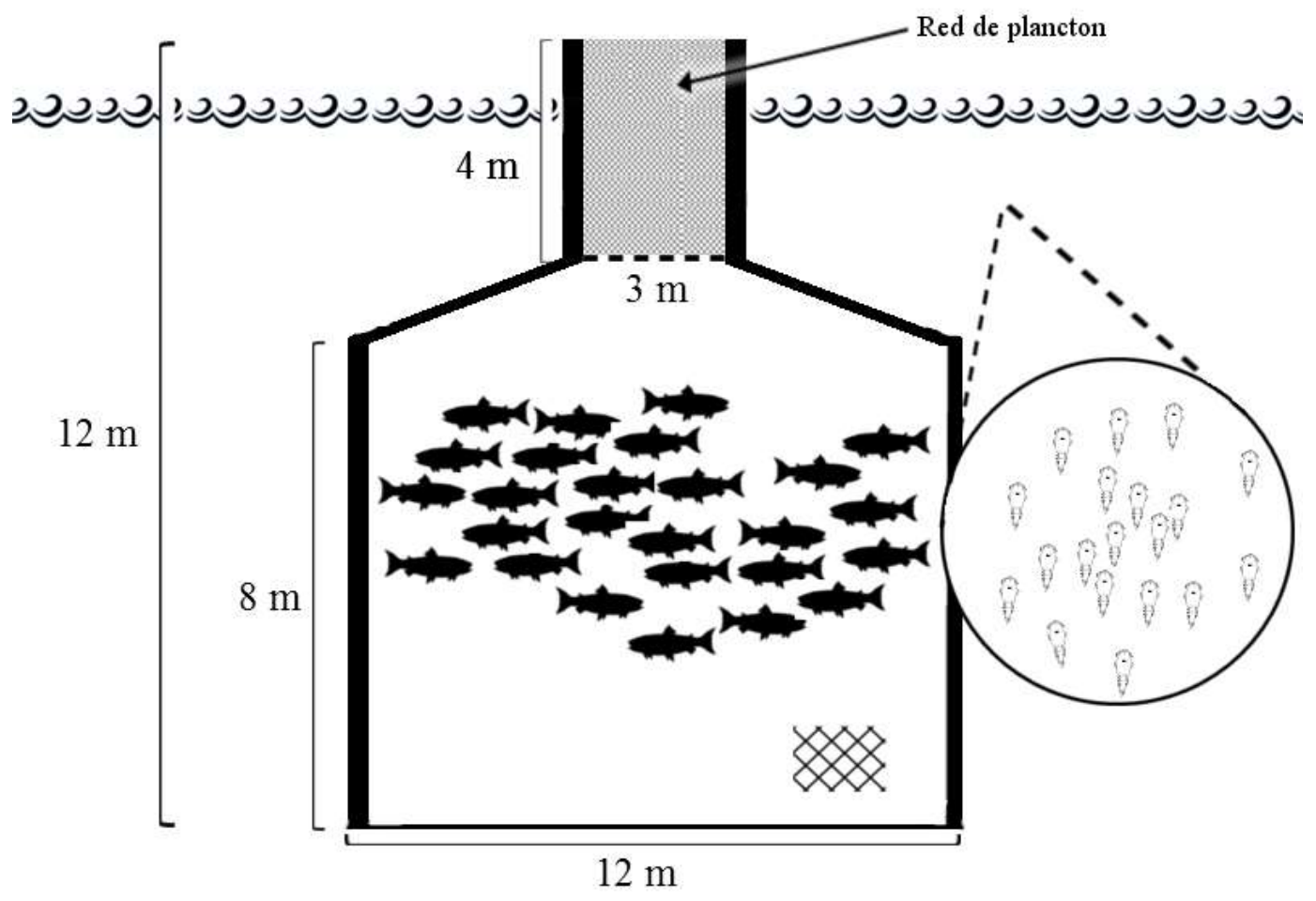

Figura 3. Jaula de cultivo de peces tipo snorkel

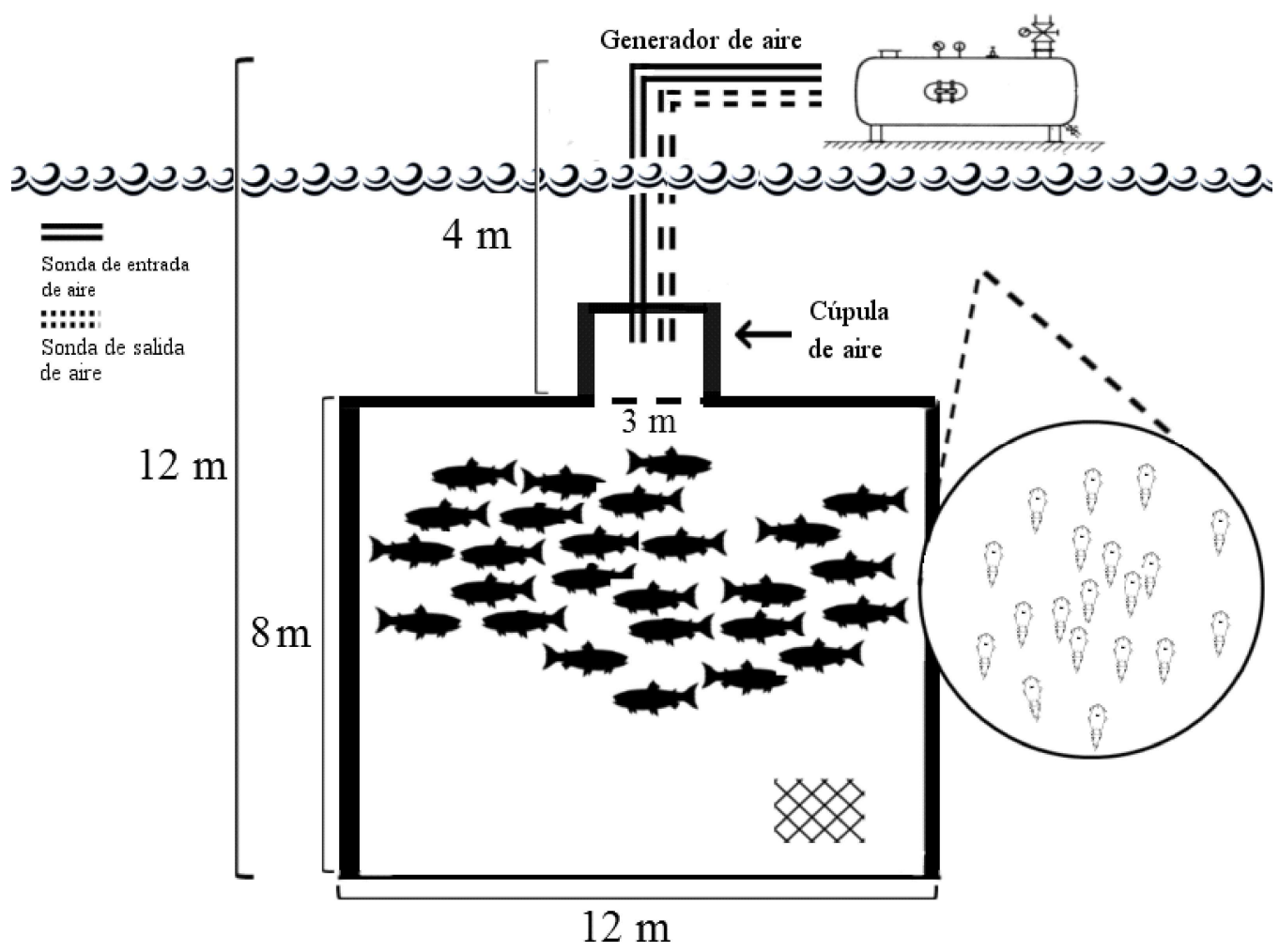

Figura 4. Jaula de cultivo de peces con cúpula de aire en su techo 
El cultivo en jaulas sumergidas presenta prometedoras ventajas en el control de condiciones ambientales fluctuantes, reduciendo, además, el contagio por C. rogercresseyi (Korsøen et al., 2009). Este tipo de cultivo presenta la desventaja de impedir al pez el acceso a la superficie para llenar de aire su vejiga natatoria (Dempster et al., 2008), de allí que se desarrolló un método para solucionar este problema, introduciendo en el techo de la jaula sumergida una cúpula llena de aire (Figura 4), lugar en el que los peces pueden llenar sus vejigas y regular su flotabilidad (Oppedal et al., 2016). El hecho de que estas jaulas permanezcan sumergidas ayuda sustancialmente a reducir los niveles de caligidosis, ya que se evita el contacto de los peces con el parásito y así los peces pueden mantener sus índices productivos (Korsøen et al., 2009).

Si bien el uso de la temperatura parece ser una variable adecuada para reducir el parasitismo sin afectar el desarrollo del cultivo de salmón, no se dispone de suficiente información para comprender el mecanismo en su totalidad. Falta discernir los efectos de UV, oxígeno, salinidad y otras variables fisicoquímicas, así como corrientes propias del posicionamiento de las jaulas, que deben ser entendidas a través de la investigación.

\section{Agradecimientos}

Jorge Parodi recibió aportes del proyecto MECESUP UCT 0804. Pamela Olivares recibió aportes del convenio de asistencia técnica UCT 278-2532 con el Laboratorio Tonalli Ltda. Roberto Riquelme recibió apoyo de tesis del Laboratorio Tonalli Ltda. para realización de su tesis de médico veterinario.

\section{Literatura Citada}

1. Bravo S. 2010. The reproductive output of sea lice Caligus rogercresseyi under controlled conditions. Exp Parasitol 125:
51-54. doi: 10.1016/j.exppara.2009. 12.001

2. Bravo S, Pozo V, Silva M, Albarca D. 2013. Comparison of the fecundity rate of Caligus rogercresseyi infesting Atlantic salmon (Salmo salar L.) on farms in two regions of Chile. Aquaculture 404-405: 55-58. doi: 10.1016/ j.aquaculture.2013.04.002

3. Bravo S, Pozo V, Silva M. 2015a. Evaluación de la efectividad del tratamiento con agua dulce para el control del piojo de mar Caligus rogercresseyi Boxshall $\&$ Bravo, 2000. Lat Am J Aquat Res 43: 322-328. doi: 10.3856/vol43-issue2fulltext-8

4. Bravo S, Silva M, Agusti C, Sambra K, Horsberg TE. 2015b. The effect of chemotherapeutic drugs used to control sea lice on the hatching viability of egg strings from Caligus rogercresseyi. Aquaculture 443: 77-83. doi: 10.1016/ j.aquaculture.2015.03.011

5. Bravo S, Silva M, Treasurer J. 2014. Factors affecting the abundance of Caligus rogercresseyi (Boxshall and Bravo) on farmed salmonids in Chile in the period 2006-2007. Aquaculture 434: 456-461. doi: 10.1016/j.aquaculture. 2014.09.009

6. Bricknell I, Dalesman S, O'Shea B, Pert C, Mordue A. 2006. Effect of environmental salinity on sea lice Lepeophtheirus salmonis settlement success. Dis Aquat Organ 71: 201-212.

7. Dempster T, Korsoen O, Folkedal O, Juell J, Oppedal F. 2008. Submergence of Atlantic salmon (Salmo salar L.) in commercial scale seacages: a potential short-term solution to poor surface conditions. Aquaculture 288: 254-263. doi: 10.1016/j.aquaculture. 2008.12.003

8. Farías D. 2005. Aspectos biológicos y conductuales del estadio infectante de Caligus rogercresseyi Boxshall \& Bravo 2000 (Copepoda: Caligidae), en peces nativos y de cultivo de Chile. Tesis de Biólogo Marino. Valdivia, Chile: Universidad Austral de Chile. 47 p. 
9. Fernández A, Blanco G, Vázquez E, McCarthy I, Sánchez-Prado A. 2002. Estrategias de crecimiento de una población de salmón Atlántico Salmo salar L., 1758 en condiciones ambientales diferentes. Bol Inst Esp Oceanogr 18: 363-369.

10. Fernández F. 1978. Metabolismo y alimentación en copépodos planctónicos del Mediterráneo: respuesta a la temperatura. Inv Pesq 42: 97-139.

11. Flores H, Vergara A. 2012. Efecto de reducir la frecuencia de alimentación en la supervivencia, crecimiento, conversión y conducta alimenticia en juveniles de salmón del Atlántico Salmo salar (A17 1758): experiencia a nivel productivo. Lat Am J Aquat Res 40: 536-544. doi: 103856/vol40-issue3-fulltext-3

12. Fore M, Alfredsena A, Gronningsater A. 2011. Development of two telemetrybased systems for monitoring the feeding behaviour of Atlantic salmon (Salmo salar L.) in aquaculture sea-cages. Comput Electron Agr 76: 240-251. doi: 10.1016/j.compag.2011.02.003

13. Gajardo V. 2011. Evaluación de la sobrevivencia y metamorfosis de larvas de Caligus rogercresseyi, en función de la temperatura, salinidad y oxígeno. Tesis de Ingeniería en Acuicultura. Temuco, Chile: Universidad Católica de Temuco. $67 \mathrm{p}$.

14. Gonzáles L, Carvajal J. 2003. Life cycle of Caligus rogercresseyi, (Copepoda: Caligidae) parasite of Chilean reared salmonids. Aquaculture 220: 101-117. doi: 10.1016/S00448486(02)00512-4

15. González L, Carvajal J, GeorgeNascimiento M. 2000. Differential infectivity of Caligus flexispina (Copepoda, Caligidae) in three farmed salmonids in Chile. Aquaculture 183: 1323. doi: 10.1016/S0044-8486(99)00282-3

16. González M, Ortiz B, González M. 2001. Saprolegniasis in wild fish populations. Cienc Marinas 27: 125-137.
17. Jonhson S, Albright L. 1992. Comparative susceptibility and histopathology of the response of naive Atlantic, chinook and coho salmon to experimental infection with Lepeophtheirus salmonis (Copepoda: Caligidae). Dis Aquat Organ 14: 179193. doi: 10.3354/dao014179

18. Korsøen O, Dempster T, Fjelldal P, Oppedal F, Kristiansen T. 2009. Longterm culture of Atlantic salmon (Salmo salar) in submerged cages during winter affects behaviour, growth and condition. Aquaculture 296: 373-381. doi: 10.1016/ j.aquaculture.2009.08.036

19. Korsoen O, Dempster T, Fosseidengen J, Ferno A, Heegaard H, Kristiansen T. 2010. Behavioural responses to pressure changes in cultured Atlantic cod (Gadus morhua): defining practical limits for submerging and lifting sea-cages. Aquaculture 308: 106-115. doi: 10.1016/j.aquaculture. 2010.08 .011

20. Korsoen $O$, Fosseifengen $J$, Kristiansen S, Oppedal F, Bui S, Dempster T. 2011. Atlantic salmon ( $\mathrm{Sal}$ mo salar L.) in a submerged sea-cage adapt rapidly to re-fill their swim bladders in an underwater air filled dome. Aquacult Eng 51: 1-6. doi: 10.1016/ j.aquaeng.2012.04.001

21. Lonsdale D, Frey M, Snell T. 1998. The role of chermical signals is copepod reproduction. J Mar Sys 15: 1-12. doi: 10.1016/S0924-7963(97)00044-4

22. Nieto D, Norambuena $R$, Gonzáles $E$, Gonzáles L, Bett D. 2010. Sistemas de producción de smolts en Chile. Análisis de alternativa desde la perspectiva ambiental, sanitaria y económica. Valdivia, Chile: WWF. 40 p.

23. Nytro A, Vikingstad E, Foss A, Hangstand T, Reynolds P, Eliassen G, Elvegaird T, et al. 2013. The effect temperature and fish size on growth of juvenile lumpfish (Cyclopterus lumpus L.). Aquaculture 434: 296-302. doi: 10.1016/j.aquaculture.2014.07.028 
24. Oppedal F, Dempster T, Stien L. 2016. Snorkelmerd: Produksjonseffektivitet, adferd og velferd. Report No. 9. Institute of Marine Research. 37 p.

25. Osorio V. 2006. Conducta de apareamiento y reproducción del ectoparásito Caligus rogercresseyi (Boxshall \& Bravo, 2000) (Copepoda: Caligidae) en el hospedador Eleginops maclovinus (Valenciennes, 1840) (Piscies: Nototheniidae). Osorno, Chile: Universidad de los Lagos [Internet]. Disponible en: http:// www.i-mar.cl/investigacion/tesis/ TESIS\%20VERO\% 20OSORIO. pdf

26. Ritchie G, Morgue A, Pike A, Rae G 1996. Observation on mating and reproductive behavior of Lepeophtheirus salmonis, Kroger (Copepoda: caligidae). J Exp Mar Biol Ecol. 201: 285-298.

27. Rodríguez M, Rodríguez G, Monroy Y, Mata JA. 2001. Manual de enfermedades de peces. Bol Programa Nacional de Sanidad Acuícola y Red de Diagnóstico 3(13): 1-14.

28. SHOA. 2015. Temperatura del agua de mar. In Servicio Hidrográfico y Oceanográfico de la Armada. (Santiago). [Internet]. Disponible en: http://www. shoa.cl/nuestros-servicios/tsm

29. Silva G 2007. Salmónidos 2006: volando alto. Revista Aqua 114: 6-13.
30. Solis A. 2009. Sistema de control de inventario de peces vivos para la industria salmonicultura. Tesis de Magíster. Santiago de Chile: Universidad de Chile. $114 \mathrm{p}$.

31. Stien L, Dempster T, Bui S, Glaropoulos A, Fosseidengen J, Wright D, Oppedal F. 2015. 'Snorkel' sea lice barrier technology reduces sea lice loads on harvest-sized Atlantic salmon with minimal welfare impacts. Aquaculture 458: 29-37. doi: 10.1016/ j.aquaculture.2016.02.014

32. Vanderstichel $R$, St-Hilaire S, Ibarra $R$, Lyngstad M, Rees E, Medina $M$. 2014. Space-time cluster analysis of the non-pathogenic infectious salmon anemia virus (HPR0 ISAV) in Chile, 2011-2012. Aquaculture 437: 120-126. doi: 10.1016/ j.aquaculture.2014.11.027

33. Wijekoon M, Parrish C, Mansour A. 2014. Effect of dietary substitution of fish oil with flaxseed or sunflower oil on muscle fatty acid composition in juvenile steelhead trout (Oncorhynchus mykiss) reared at varying temperatures. Aquaculture 433: 74-81. doi: 10.1016/ j.aquaculture.2014.05.028

34. Yatabe T, Arriagada G, HamiltonWest C, Urcelay S. 2011. Risk factor analysis for sea lice, Caligus rogercresseyi, levels in farmed salmonids in southern Chile. J Fish Dis 34: 345-354. doi: 10.1111/j.1365-2761.2011.01238.x 